Sharif University of Technology
Scientia Iranica
Transactions E: Industrial Engineering
hCIENTIA

\title{
A bi-objective hierarchical hub location model with facility failure
}

\author{
M. Babashahi, K. Shahanaghi, M.R. Gholamian*, and A. Yavari \\ School of Industrial Engineering, Iran University of Science and Technology, Tehran, Iran.
}

Received 12 October 2017; received in revised form 12 September 2018; accepted 10 December 2018

\section{KEYWORDS}

Hierarchical hub location;

Backup facility;

Reliability;

Hub failure,

NSGA-II.

\begin{abstract}
In most past studies on Hub Location Problem (HLP), failure probability is limited to a constant value; however, in reality, it depends on various factors including natural disasters such as floods and earthquakes, commodity flow, institutional elements (strikes), etc. This paper looks into the problem of the hub network design through a new model. The problem is to maximize network reliability and minimize the total routing cost simultaneously. In order to provide a more realistic model, the hub's failure probabilities were considered as a function of the hub's incoming traffic. Reserve hub elements were also taken into account in the model with the aim of increasing network reliability. To solve the model, a Non-dominated Sorting Genetic Algorithm (NSGA-II) was presented. The parameters of the algorithm were tuned using Taguchi method. The proposed solution was then carried out in a case study where the results confirmed the acceptable performance of the model; moreover, sensitivity analysis was performed in order to describe the effects of critical parameters on objective functions.
\end{abstract}

(C) 2020 Sharif University of Technology. All rights reserved.

\section{Introduction}

The decisions about the location of hubs and the allocation of demand nodes to hubs are strategic decisions that require heavy investments because a system should be used in anticipation of many coming years under these conditions [1]. The overall performance of a network extremely depends on the proper performance of its underlying elements (hubs and arcs). The failure of one element (hubs or arcs) may lead to the network breakdown or poor service levels. Therefore, the priority is to design networks with higher reliability. Reliability is defined as the probability that a facility (hub and arcs) or a route performs its operations

*. Corresponding author. Tel.: +9821 7322 5067 E-mail address: Gholamian@iust.ac.ir (M.R. Gholamian)

doi: $10.24200 /$ sci. 2018.5358 .1229 flawlessly over a time period without failure, delay, or congestion (e.g., transmitting flows over a time period without failure, delay, or congestion) [2].

Kim and O'Kelly [2] considered the concept of reliability in HLP for the first time and presented a reliable p-HLP that focuses on maximizing network performance in terms of reliability by locating hubs for delivering flows among city nodes. In their study, the relationship between network performance and hub facility location by using telecommunication networks in the United States was explored. Davari et al. [3] designed a single-allocation hub location so that the reliability of the network could be maximized. Fazel et al. [4] presented a new method for obtaining the reliability of paths in a hub-and-spoke network and, then, designed a single-allocation hub location by maximizing the weighted reliability of the network as its first objective and maximizing minimum reliability in the network as its second objective. Aiming to achieve the 
minimum cost of the network establishment, Karimi and Bashiri [5] provided a model that ensures the reliability of paths that pass through one hub in not less than a specified threshold value. However, their study considered the reliability of arcs only for determining the reliability of paths. Recently, Hamidi et al. [6] developed a new type of reliability called "prevention reliability" that makes the network reliable by adding fake items to it.

One of the famous variants of HLP includes hierarchical hub networks, among many other types of networks. Yaman [7] is one of the first researchers in this field. She considered the problem of designing a three-level hub network with the following structure; the central hubs were connected in a complete network at the top level, and the remaining hubs and demand centers were connected to the central hubs through star networks at the second and third levels, respectively. Hence, a hierarchical network structure was formed. Among recent examples in this area, one can point to the study of Hamzaoui and Ben-Ayed [8] that developed an effective time-tabling program in a hierarchical HLP of the parcel distribution network. A star/star two-level HLP model for telecommunication networks was developed by Yaman and Elloumi [9], which minimized the longest path at the first level and routing cost at the second level. Meanwhile, two algorithms were developed for solving the models. Alumur et al. [10] introduced a hierarchical multimodal HLP for a Turkish cargo delivery network. They supposed two transportation modes: a highway segment and an airline segment at the levels of hierarchy structure. Davari and Fazel [11] extended Yaman's model [7] with fuzzy variables. The model was developed based on credibility measures. Moreover, Fazel et al. [12] developed two meta-heuristics for hierarchical HLP and compared the results. The application of capacitated hierarchical HLP model for public transportation between urban and rural areas was developed by Zhong et al. [13]. The model was solved using a hybrid genetictabu search optimization algorithm. In addition, in the case of healthcare systems, Smith et al. [14] developed a hierarchical HLP model for the network of laboratories, which are responsible for blood sample collection for HIV/AIDS. They developed two mixed integer programming models so that the total number of laboratories could be minimized in the first model and the total travel time could be minimized in the second model. The models were run in the real case study in South Africa, and the results were compared with the current situation. Sedehzadeh et al. [15] applied both fuzzy modeling and meta-heuristics solution approach. The model seeks to minimize cost and fuel consumption simultaneously by considering various modes of transportation in a tree hierarchical HLP. It was assumed that the costs, capacities, and speed of vehicles were uncertain and, therefore, a fuzzy programming with triangular numbers was developed for the model. Finally, the model was solved with two multi-objective meta-heuristics (i.e., NSGA-II and Multi-Objective Imperialist Competitive Algorithm (MOICA)).

Another extension was proposed by Korani and Sahraeian [16]. They developed a hierarchical hub covering problem where the nodes were assigned to hubs based on cover radii. $\mathrm{Li}$ et al. [17] developed a hierarchical HLP for passenger hub layout by considering both hub coverage and hub number of urban agglomerations. They extended their work to a multiperiod model and solved the model using a variation of adaptive clonal selection algorithm [18]. Moreover, recently, Dükkanci and Kara [19] introduced a hierarchical multimodal hub covering problem, whereas the upper level was configured by ring structure instead of star structure, which is used at middle and lower levels. Therefore, the routing and scheduling constraints were added to the model. The model was solved by subgradient based heuristics and implemented on the cargo deliver application in Turkey as a case study.

On the other hand, Ryerson and Kim [20] established a hub hierarchy using machine learning techniques. Specifically, they developed triple hub clustering indices and, then, applied the k-means clustering method to data from the U.S. airline network. Karimi et al. [21] proposed a capacitated hierarchical HLP model, which is in fact the extension of previous capacitated models to hierarchical structure. Refreshment operation at different levels of the hierarchical HLP is another concept, which was introduced for perishable items by Esmizadeh and Bashiri [22]. Da Costa Fontes and Goncalves [23] presented a hierarchical HLP model through the multi-allocation of sub-hubs. They developed their work in the case of a liner shipping network with deep/shallow sea operations. In this work, the application of sub-hub linkages creates alternative paths that allow multiple allocations. Mahmutogullari and Kara [24] introduced a bi-level model for competitive hub location in the duopoly market in which each player decides on the hub location; in specific terms, the medianoid model at the first level and centroid model at the second level were used to reach the maximum amount of market share. Kim et al. [25] developed a two-layered hierarchical network to determine the hurricane evacuation routes to safe areas. They developed a model that encapsulates four cases of corridor line generation (with/without contraflow); then, the models were solved using a rule-based multiagent simulation method. The model was applied for Haeundae Beach, Busan, Korea to prevent Tsunami disasters. Torkestani et al. [26] used a dynamic system approach in a hierarchical HLP considering different probabilities of disruption at each level and each time 
period of the model. The model was solved using Monte Carlo simulation approach.

As shown earlier, in the previous works, the failure has been either assumed constant or modeled by a Bernoulli random variable, i.e., facility $i$ fails with probability $p_{i}$. In reality, however, the failure depends on various factors including natural disasters and practical disruptions.

In the model proposed here, a hierarchical threelevel hub network is considered. At the top level, the central hubs are connected within a complete network. At the second level, the remaining hubs are connected to central hubs within star networks. Finally, at the third level, the demand centers are connected to the hubs through star networks. In this model, the probability of a hub facility failure is considered to be dependent on both the commodity flow and natural effects. The dependency on the flow is assumed to be linear; therefore, the probability of hub failure is a continuous function that changes at different levels. In order to increase the reliability of the total network, for each central hub, back-up components are provided. These backup components can replace the faulty elements within each hub to prevent failure.

Based on the above descriptions, the remainder of the paper is structured as follows. Section 2 introduces the proposed model as a bi-objective mathematical programming model. Section 3 provides numerical examples and analytical results; finally, Section 4 presents conclusions and future studies.

\section{The proposed model}

In this section, the model introduced by Yaman [7] is considered as the reference model. In what follows, the model is described in more detail.

\subsection{Assumptions and conditions}

The model is open to the following assumptions:

- The network is considered at three levels;

- The number of hubs and central hubs is assumed to be $p$ and $p_{0}$, respectively;

- A single assignment scheme is allowed for the network;

- The central hubs network is defined with a full connection;

- The demand centers and the hubs are connected to the central hubs through star networks;

- The demand centers are connected to the hubs through star networks;

- Hub failures are assumed to be independent;

- The hub failure depends on external elements and on its entering flow;
- The demands at the demand centers are not completely fulfilled.

\subsection{The major decisions}

Major decisions in the model are made to determine the following:

- The location of the hubs and the central hubs;

- The traffic in the network links;

- The number of backup components at each hub.

\section{Definitions and notations}

Parameters:

- $I$ : The set of nodes;

- $H \subseteq I$ : The set of available locations for the hubs;

- $C \subseteq I$ : The set of available locations for the central hubs;

- $d_{i j}$ : The routing cost of unit traffic from node $i \in I$ to node $j \in I\left(d_{i j}=d_{j i} ; d_{i i}=0\right)$;

- $\alpha_{H}$ : The discount factor for the routing cost from the hubs to the central hubs;

- $\alpha_{C}$ : The discount factor for the routing cost among the central hubs;

- $p$ : The number of hubs that needs to be located and opened;

- $p_{0}$ : The number of central hubs that needs to be located and opened;

- $\lambda_{j}$ : The probability of failure for hub $j$ due to external effects;

- $M$ : A very large number;

- $f_{k}^{B}$ : The fixed cost of one backup component $B$ in hub $k$;

- $C a_{j}$ : The capacity of the $j$ th hub.

\section{Intermediate decision variables:}

- $t_{i m}$ : The traffic flowing from node $i \in I$ to node $m \in I$;

- $O_{j}$ : The inward (outward) flow of hub $j$;

- $P_{j}$ : The probability of flow-enforced failure for hub $j$;

- $R R_{j}$ : The probability that hub $j$ does not fail;

- $F R_{j}$ : The failure probability for hub $j$;

- $T R_{j}$ : The total failure probability for hub $j$, taking into account the backup components.

\section{Final decision variables}

- $g_{j l}^{i}$ : The traffic of node $i \in I$ as the origin or destination nodes, which flows between hub $j \in H$ and the central hub $l \in C$; 
- $f_{k l}^{i}$ : The traffic of node $i \in I$ as an origin node, which flows between the central hub $k \in C$ and the central hub $l \in C \backslash\{k\}$;

- $z_{i j l}$ : is equal to 1 if node $i \in I$ is assigned to hub $j \in H$ and hub $j$ is assigned to the central hub $l \in C$; otherwise, it is equal to 0 ;

- $x_{k}^{B}$ : The number of backup components for hub $k$.

Now that the parameters and notations are introduced, the developed model is presented in the following:

$$
\begin{aligned}
\min \sum_{i \in I} & \sum_{m \in I}\left(t_{i m}+t_{m i}\right) \sum_{j \in H} d_{i j} \sum_{l \in C} Z_{i j l} \\
& +\sum_{i \in I} \sum_{j \in H} \sum_{l \in C \backslash\{j\}} \alpha_{H} d_{j l} g_{j l}^{i} \\
& +\sum_{i \in I} \sum_{j \in H} \sum_{l \in C \backslash\{j\}} \alpha_{C} d_{j l} f_{j l}^{i}+\sum_{k \in C} f_{k}^{B} x_{k}^{B},
\end{aligned}
$$

$\sum_{j \in H} \sum_{l \in C} z_{i j l}=1 \quad \forall i \in I$

$z_{i j l} \leq z_{j j l} \quad \forall i \in I, \quad j \in H \backslash\{i\}, \quad l \in C$,

$\sum_{m \in H} z_{j m l} \leq z_{l l l} \quad \forall j \in H, \quad l \in C \backslash\{j\}$,

$\sum_{j \in H} \sum_{l \in C} z_{j j l}=p$

$\sum_{l \in C} z_{l l l}=p_{0}$

$\forall i \in I, \quad j \in H, \quad l \in C \backslash\{j\}$,

$\sum_{k \in C \backslash\{l\}} f_{l k}^{i}-\sum_{k \in C \backslash\{l\}} f_{k l}^{i}=\sum_{m \in I} t_{i m} \sum_{j \in H}\left(z_{i j l}-z_{m j l}\right)$

$\forall i \in I, \quad l \in C$,

$O_{j} \geq \sum_{i \in I} \sum_{l \in C} g_{j l}^{i}-M\left(1-\sum_{l \in C \backslash\{j\}} z_{j j l}\right)$

$\forall j \in H$,

$$
O_{j} \geq \sum_{i \in I} \sum_{k \in C} f_{j k}^{i}-M\left(1-z_{j j j}\right) \quad \forall j \in C,
$$

$$
\begin{aligned}
& P_{j}=1-\min \left\{1, \max \left\{0, \frac{O_{j}-C a_{j}}{C a_{j}}\right\}\right\} . \quad \forall j \in H, \\
& R R_{j}=\left(1-P_{j}\right)\left(1-\lambda_{j}\right) \\
& \forall j \in H, \\
& F R_{j}=\left(1-R R_{j}\right) \\
& \forall j \in H, \\
& T R_{j}=\left(1-F R_{j}^{x_{B}+1}\right) \\
& \forall j \in H \\
& x_{B} \in\{0,1,2,3\}, \\
& z_{l j l}=0 \quad \forall j \in H, \quad l \in C \backslash\{j\}, \\
& g_{j l}^{i} \geq 0 \quad \forall i \in I, \quad j \in H, \quad l \in C, \\
& f_{k l}^{i} \geq 0 \quad \forall i \in I, \quad k \in C, \quad l \in C \backslash\{j\}, \\
& t_{i m} \geq 0 \quad \forall i \in I, \quad m \in I, \\
& O_{j}, P_{j}, R R_{j}, F R_{j}, T R_{j} \leq 0 \quad \forall j \in H, \\
& z_{i j l} \in\{0,1\} \quad \forall i \in I, \quad j \in H, \quad l \in C .
\end{aligned}
$$

In this model, the first objective function (1) illustrates the backup establishment cost plus the costs of all the possible routing traffic between the nodes, including the traffic between demand nodes and their hub, between the hubs and their central hubs, and among central hubs. In contrast, the second objective function (2) maximizes the lowest level of network reliability.

Constraint (3) guarantees that each demand node is assigned to a hub or a central hub. Constraint (4) states that if node $i$ is assigned to hub $j$ and central hub $l$, then node $j$ should be a hub assigned to the central hub $l$. According to Constraint (5), if node $j$ is assigned to the central hub $l$, then Hub $l$ must be a central hub. Constraints (6) and (7) reflect the fixed number of hubs and central hubs to be opened, i.e., $p$ and $p_{0}$, respectively. The values of $g_{j l}^{i}$ in terms of the assignment variables are determined by Constraints (8) and (18). Constraint (9) balances the flow between nodes in the hierarchy. The already introduced constraints are approximately the same as the ones used in the basic model. In the following, new constraints are introduced.

Constraints (10) and (11) demonstrate the incoming flow in the $j$ th and $l$ th hubs, respectively. Constraint (12) gives the probability of failure for the lst hub in terms of its incoming flow. If the incoming flow to this hub exceeds the maximum tolerable level, this probability increases to 1 and the hub will fail. This constraint can be converted into a set of linear constraints by adding new variables, as shown in 
Eqs. (23) and (24):

$$
\begin{aligned}
& v=\max \left(0, \frac{O_{j}-C a_{j}}{C a_{j}}\right) \Rightarrow v \geq \frac{O_{j}-C a_{j}}{C a_{j}} \quad \& \quad v \geq 0 \\
& u=\min (1, v) \Rightarrow u \leq v \quad \& \quad u \leq 1
\end{aligned}
$$

Constraint (13) gives the functioning probability of the lth hub. Constraint (14) provides the probability of total failure for the lst hub. Constraint (15) determines the functioning probability of the lst hub considering the backup elements. Constraints (16)-(22) represent integrality and non-negativity of variables. However, it should be noted that the redundant Constraints (19) could further strengthen the Linear Programming (LP) relaxation.

The proposed model in this paper is a nonlinear model. In order to reduce the nonlinearity of the model, a heuristic with acceptable approximation is proposed. For this reason, first, the range $[0,1]$ is partitioned logarithmically into partitions indexed by $n=1,2, \ldots, 10$. Each of these values contains four cases based on the range of $x_{j}^{B}$. Consider the following new parameters:

$a_{n t} \quad$ the approximate number of values that falls in partition $n$ with a power of $t$;

$y_{j n} \quad$ is equal to 1 if the approximate $F R_{j}$ falls in the $n$th partition; otherwise, it is equal to 0 ;

$x_{j t} \quad$ is equal to 1 if $\mathrm{t}$ backup facilities are deployed in the $j$ th hub; otherwise, it is equal to 0 .

Now, the following constraints can be added to the model:

$$
\begin{aligned}
& F R_{j} \leq \sum_{n} a_{n 1} y_{j n} \quad \forall j \in H, \\
& T R_{j} \leq 1-\sum_{t} a_{n t} x_{j t}^{B}+M\left(1-y_{j n}\right) \quad \forall j \in H, n, \\
& \sum_{t} x_{k t}^{B}=1 \quad \forall k \in C .
\end{aligned}
$$

Constraint (25) demonstrates the approximate value of $F R_{j}$. The value of $y_{j n}$ equals 1 only for one value of $n$; otherwise, it is equal to 0 ; therefore, $a_{n 1}$ corresponding to each $F R_{j}$ is obtained through this constraint. Constraint (26) computes the approximate value of $T R_{j}$. Constraint (27) ensures that a certain number of backup elements are assigned to each central hub. Moreover, the objective function (1) can be rewritten as follows:

$$
\begin{aligned}
\min \sum_{i \in I} & \sum_{m \in I}\left(t_{i m}+t_{m i}\right) \sum_{j \in H} d_{i j} \sum_{l \in C} z_{i j l} \\
& +\sum_{i \in I} \sum_{j \in H} \sum_{l \in C \backslash\{j\}} \alpha_{H} d_{j l} g_{j l}^{i} \\
& +\sum_{i \in I} \sum_{j \in H} \sum_{l \in C \backslash\{j\}} \alpha_{C} d_{j l} f_{j l}^{i} \\
& +\sum_{k \in C}(t-1) f_{k}^{B} x_{k t}^{B} .
\end{aligned}
$$

The bi-objective model has been solved using $\varepsilon$-constraint method [27]. To do so, the first objective function is restricted to the bounds $(L)$, which are defined in the range of the worst $\left(T C^{D}\right)$ to the best $\left(T C^{U}\right)$ values of this objective function considering predefined step size $(r)$, as shown in Eq. (29):

$$
\begin{aligned}
& L_{n}=T C^{D}+\frac{n}{r-1}\left(T C^{U}-T C^{D}\right) \\
& n=0,1,2, \cdots, r-1 .
\end{aligned}
$$

Then, the model is converted into a single-objective one by considering the first objective function as a constraint, as shown in Eq. (30):

$$
\begin{gathered}
\sum_{i \in I} \sum_{m \in I}\left(t_{i m}+t_{m i}\right) \sum_{j \in H} d_{i j} \sum_{l \in C} z_{i j l} \\
\quad+\sum_{i \in I} \sum_{j \in H} \sum_{l \in C \backslash\{j\}} \alpha_{H} d_{j l} g_{j l}^{i} \\
\quad+\sum_{i \in I} \sum_{j \in H} \sum_{l \in C \backslash\{j\}} \alpha_{C} d_{j l} f_{j l}^{i} \\
\quad+\sum_{k \in C}(t-1) f_{k}^{B} x_{k t}^{B} \leq L_{n} .
\end{gathered}
$$

Meanwhile, the second objective function can be simplified by considering:

$$
\max : T R_{m}=\min _{j} T R_{j}
$$

and adding a new set of constraints to the model, as shown in Eq. (32):

$$
T R_{j} \geq T R_{m}
$$

Solving this single-objective model in each $n=0,1,2, \cdots, r-1$ will generate the plot of Pareto frontier, as shown in the next section. 


\section{Numerical results}

Now that the model is mathematically described, a number of solutions are given for the proposed model. For small-scale problems, the problem is solved via the LINGO - CPLEX solver. As for the problems on larger scales, the NSGA-II is used where Taguchi method is applied to obtain the optimal parameters of the model.

In order to show the efficiency of the proposed solution, the problem is solved in low dimensions using a CPLEX solver and, then, the results are compared with those obtained by NAGA-II. Finally, the model is verified by performing sensitivity analysis of the critical parameters of the model.

The computational experiments are carried out on a PC characterized by Windows 7, version 2012, and Core-I 5, 2.5 GHz CPU with 4 Giga bytes of RAM.

\subsection{The case study}

The case study considered in this paper is based on a real-life postal network with 20 nodes, whose data were reported by Ernest and Krishnamurthy [28]. Since there is no complete database available in the literature, a random data generator is used for the missing parameters. The failure probabilities due to natural effects are considered independently and identically distributed over [0.2 0.3$]$, according to a uniform distribution. Moreover, the cost for each substitute component is assumed to be random and uniformly distributed over [15000, 20000].

In Table 1 , the outputs of $N=20, p_{1}=5$ along with different values of $\alpha_{C}$ and $\alpha_{H}$ are shown. Note that in problems of larger dimension, global optimization with Operations Research (OR) applications such as LINGO is impossible to achieve. Meanwhile, some heuristics such as Benders' decomposition have been examined. However, unfortunately, no results have been achieved for large-scale problems and, thus, we are forced to solve these problems with meta-heuristics such as NSGA-II.

As a sample, Figure 1 depicts the hub and central hub location assignments for $\left(\alpha_{C}, \alpha_{H}\right)=(1,1)$, i.e., the first line of Table 1.

As observed in Figure 1, nodes 10, 14, 20, 7, and 11 are chosen as hubs, and node 10 is chosen as the central hub. Notice that $X$ and $Y$ in Figure 1 are the

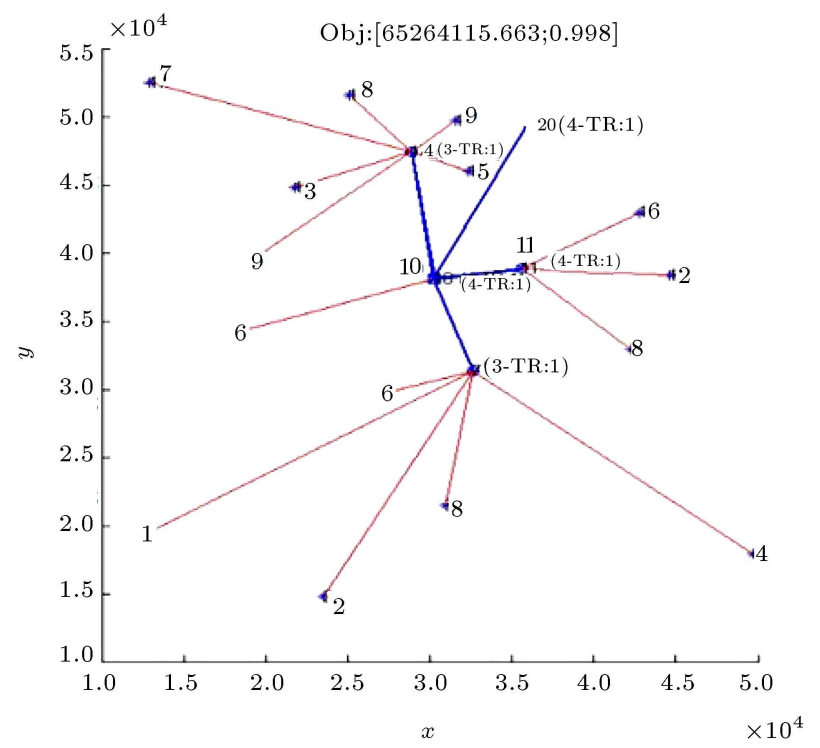

Figure 1. The hub and central hub location assignments for $N=20$ and $P_{1}=5$.

Table 1. The hubs and central hubs assignment for $N=20$ and $P_{1}=5$.

\begin{tabular}{|c|c|c|c|c|c|c|}
\hline$\left(\alpha_{C}, \alpha_{H}\right)$ & $P_{2}$ & $\begin{array}{c}\text { Central hubs } \\
(\text { GA) }\end{array}$ & $\begin{array}{l}\text { Hubs } \\
\text { (GA) }\end{array}$ & Objective 1 & Objective 2 & $\begin{array}{c}\text { CPU time } \\
(\mathrm{GA})\end{array}$ \\
\hline \multirow{3}{*}{$(1,1)$} & 1 & 10 & $10,14,20,7,11$ & $6.52 \mathrm{e}+7$ & 0.9985 & 15019 \\
\hline & 3 & $10,15,14$ & $13,11,15,14,10$ & $6.21 \mathrm{e}+7$ & .0 .9985 & 5201 \\
\hline & 4 & $10,15,14,11$ & $11,15,14,10,13$ & $6.01 \mathrm{e}+7$ & .9985 & 4926 \\
\hline \multirow{3}{*}{$(0.9,0.9)$} & 2 & 10,6 & $14,7,11,6,10$ & $6.39 \mathrm{e}+7$ & 0.9985 & 4914 \\
\hline & 3 & $15,10,6$ & $10,6,15,14,18$ & $6.01 \mathrm{e}+7$ & 0.9985 & 5506 \\
\hline & 4 & $15,10,11,6$ & $6,14,11,15,10$ & $5.98 \mathrm{e}+7$ & 0.9985 & 4929 \\
\hline \multirow{3}{*}{$(0.8,0.8)$} & 2 & 15,10 & $14,6,10,15,11$ & $6.26 \mathrm{e}+7$ & 0.9985 & 4941 \\
\hline & 3 & $19,14,15$ & $15,11,14,10,19$ & $5.72 \mathrm{e}+7$ & 0.9985 & 4980 \\
\hline & 4 & $14,19,15,10$ & $6,10,15,19,14$ & $5.65 \mathrm{e}+7$ & 0.9985 & 4661 \\
\hline \multirow{3}{*}{$(0.9,0.8)$} & 2 & 19,15 & $14,11,19,20,15$ & $6.27 \mathrm{e}+7$ & 0.9985 & 5245 \\
\hline & 3 & $19,15,14$ & $20,19,15,11,14$ & $6.12 \mathrm{e}+7$ & 0.9985 & 4964 \\
\hline & 4 & $15,19,10,14$ & $19,7,15,14,10$ & $6.015 \mathrm{e}+7$ & 0.9985 & 5582 \\
\hline
\end{tabular}


distances of locations from each other. Figure 2 further shows the Pareto solution set for the studied problem. The represented result is obtained by the $\varepsilon$-constraint method. The bounds of the first objective function are considered in the range $[6.4956 .527] \times 10^{7}$ with step size 20,000, as shown in Table 2. The spacing metric suggested by Schott [29] is also calculated in this table.

In Table 2, the first and second columns represent the optimal Pareto solutions obtained from solving the model using $\varepsilon$-constraint method. The third column represents the relative distance measure between con-

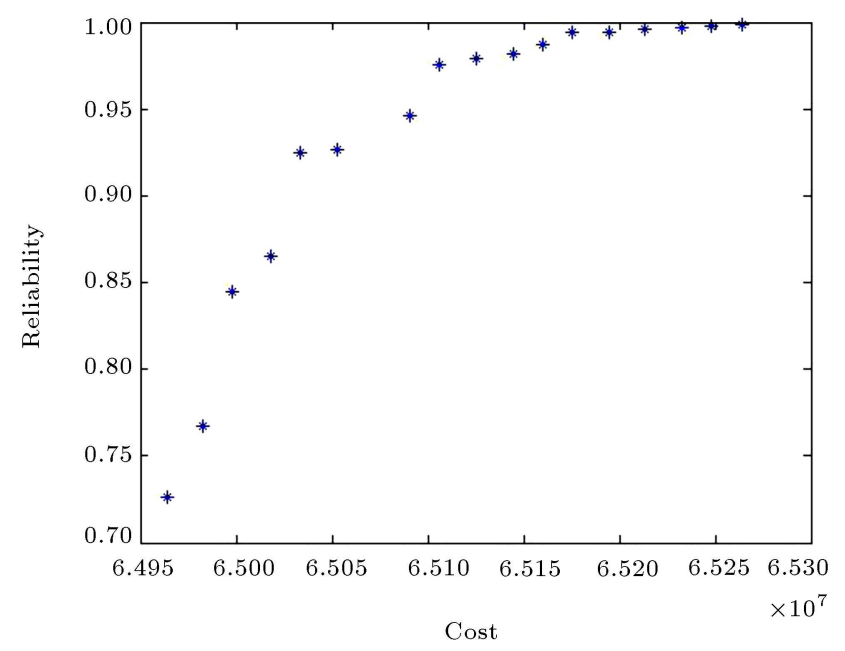

Figure 2. The Pareto solution for $N=20$ and $P_{1}=5$.

Table 2. The results of running $\varepsilon$-constraint method.

\begin{tabular}{ccc}
\hline Cost $\left.\mathbf{( 1 0}^{\mathbf{7}}\right)$ & Reliability & $\mathbf{d}(\mathbf{\%})$ \\
\hline 6.495 & 0.726 & 4.1 \\
6.497 & 0.767 & 4.1 \\
6.499 & 0.847 & 1.6 \\
6.501 & 0.863 & 1.6 \\
6.503 & 0.922 & 0.3 \\
6.505 & 0.925 & 0.3 \\
6.507 & 0.947 & 2.2 \\
6.509 & 0.976 & 0.4 \\
6.511 & 0.98 & 0.2 \\
6.513 & 0.982 & 0.2 \\
6.515 & 0.989 & 0.7 \\
6.517 & 0.996 & 0 \\
6.519 & 0.996 & 0 \\
6.521 & 0.997 & 0.1 \\
6.523 & 0.998 & 0.1 \\
6.525 & 0.999 & 0.1 \\
6.527 & 1 & 0.1 \\
Spacing metric $=$ & 1.31244 \\
\hline
\end{tabular}

Table 3. Number of reserve elements for each hub.

\begin{tabular}{cc}
\hline No. of reserved elements & Number of hub \\
\hline 4 & 10 \\
3 & 14 \\
4 & 20 \\
3 & 7 \\
4 & 11 \\
\hline
\end{tabular}

secutive Pareto optimal solutions $\left(d_{i}\right)$, as shown in Eq. (33):

$$
d_{i}=\min _{k \in N_{i}}\left\{Z_{i}-Z_{k}\right\}
$$

where $N_{i}$ is a set of Pareto solutions close to the point $\mathrm{i}$, and $Z_{i}$ is the optimal objective vector at $i$.

The last row represents a spacing metric calculated based on Euclidean distance of $d_{i}$ 's from the mean through Eq. (34):

$$
S=\sqrt{\frac{1}{n} \sum_{i=1}^{n}\left(d_{i}-\bar{d}\right)^{2}}
$$

where $n$ is the number of Pareto solutions, and $\bar{d}$ represents the mean of distance measures.

In Figure 2, the reliability of the total network changes in an acceptable range. In Table 3, the number of backup (reserve) elements for the hubs is shown. Moreover, in order to reach the maximum reliability, the hubs should utilize the reserve elements.

\subsection{Selecting related parameters for solving the problem}

Heuristic and Meta-Heuristic methods usually need to appropriately set some parameters in their searching process. These parameters should be set in a way that the algorithm reaches the optimal solution in the shortest possible time. The complexity of difficult problems highly depends on the size of the problem. In this paper, the size of the problem mainly depends on a few parameters including the number of demand nodes, the number of hubs, and the number of central hubs. The experiments at three size levels: small, moderate, and large. The values of the parameters are randomly chosen according to the ranges given in Table 4. At each level, 10-30 problems are considered for parameter selection, and the four main parameters involved in the solution are analyzed whose optimal levels are obtained. In order to reduce the number of experiments, Taguchi's method is carried out. Table 5 summarizes the network parameters and the NSGA-II factors at each pre-defined level.

Finally, each problem is named according to its network parameters. For example, the problem in which the network consists of 15 demand nodes, 7 
Table 4. Network parameters for the three complexity (size) levels.

\begin{tabular}{lccc}
\hline & Small & Moderate & Large \\
\hline Number of demand nodes & $5-7$ & $7-15$ & $15-25$ \\
The number of hubs & $2-3$ & $4-7$ & $8-10$ \\
The number of central hubs & $1-2$ & $3-4$ & $5-7$ \\
\hline
\end{tabular}

Table 5. The factors involved in the solution at each complexity level.

\begin{tabular}{lccc}
\hline & Level 1 & Level 2 & Level 3 \\
\hline Population & 30 & 45 & 60 \\
Mutation rate-crossover rate & $0.3-0.7$ & $0.5-0.5$ & $0.3-0.7$ \\
Iteration & 500 & 2000 & 4000 \\
Improvement rate & 0.2 & 0.5 & - \\
\hline
\end{tabular}

hubs, and 4 central hubs is named N15_H7_C5_P1, where $P$ denotes the problem number at its relevant level.

\subsection{Problem evaluations}

As mentioned before, in order to evaluate the efficiency of the proposed algorithm, sample problems have been solved using NSGA-II and Lingo 10. Table 6 summarizes the results of the first objective. Note that the reported results of the NSGA-II method illustrate the minimum values over 5 repetitions. The results indicate the proximity of NSGA-II results to Lingo outputs with good and acceptable approximation. Although the size of the problem increases, the OR applications fail to solve the problem.

According to the results summarized in Table 6 , the performance of the proposed algorithm can be evaluated very well. Furthermore, in order to demonstrate the efficiency of the approximate linear model, smallsized problems have been solved, as shown in Table 7.
In small-size problems, the linear model gives results that are quite close to those of the nonlinear model.

\subsection{Sensitivity analysis}

In this section, the effects of critical parameters on the final results are analyzed. Specifically, the effect of the discount factor on the routing cost between the hubs and the central hubs $\left(\alpha_{C}\right)$, the discount factor on the routing cost between the central hubs $\left(\alpha_{H}\right)$, the number of central hubs $\left(p_{0}\right)$, the constant cost of one backup unit at the $k$ th hub $\left(f_{k}^{B}\right)$, and the probability of failure due to environmental effects $(\lambda)$ are studied.

Figure 3 depicts how the objective function changes with $\alpha_{H}$ and $\alpha_{C}$, respectively. As shown earlier, the cost function increases as the discount factors increase. The comparison of the trends of the curves in this figure indicates that the cost function is more sensitive to $\alpha_{H}$.

Figure 4 shows how the cost function changes with the number of central hubs and $f_{k}^{B}$. As illustrated, the

Table 6. A comparison of the results of NSGA-II and Lingo.

\begin{tabular}{ccccccc}
\hline \multirow{2}{*}{$\begin{array}{c}\text { The problem } \\
\text { (size) }\end{array}$} & \multicolumn{2}{c}{ The first objective function } & & \multicolumn{2}{c}{$\begin{array}{c}\text { The elapsed time } \\
\text { (sec) }\end{array}$} \\
\cline { 2 - 3 } & NSGA-II & Lingo & GAP & GA & Lingo \\
\hline N10_H3_C1_P1 & 14903593 & 14902354 & $8.314 \mathrm{e}-5$ & 4517 & 20 \\
N10_H5_C1_P2 & 14639385 & 14617236 & 0.0015 & 4446 & 129 \\
N10_H5_C2_P3 & 15286284 & 15262153 & & 0.0016 & 4870 & 10542 \\
N15_H3_C1_P4 & 36919222 & 36400128 & & 0.0143 & 4926 & 65423 \\
N15_H5_C2_P5 & 36329565 & - & - & 4953 & - \\
N15_H7_C3_P6 & 34878527 & - & - & 5019 & - \\
N15_H7_C4_P7 & 34667458 & - & - & 5169 & - \\
N20_H3_C1_P8 & 64634229 & - & - & 5260 & - \\
N20_H5_C2_P9 & 63698681 & - & - & 5290 & - \\
N20_H7_C2_P10 & 62250930 & - & - & 5384 & - \\
N20_H7_C3_P11 & 67587991 & - & - & 5688 & - \\
N20_H7_C4_P12 & 59149467 & - & - & 5800 & - \\
\hline
\end{tabular}


Table 7. A comparison of the results of the linear model and those of the nonlinear model.

\begin{tabular}{ccccccc}
\hline \multirow{2}{*}{$\begin{array}{c}\text { The problem } \\
\text { (size) }\end{array}$} & \multicolumn{2}{c}{ The objective function } & & \multicolumn{2}{c}{ The elapsed time (sec) } \\
\cline { 2 - 3 } & Nonlinear & Linear & GAP & Nonlinear & Linear \\
\hline N5_H2_C1_P1 & $1.92 \mathrm{e}+6$ & $1.99 \mathrm{e}+6$ & -0.0352 & 129 & 1 \\
N5_H3_C1_P2 & $1.66 \mathrm{e}+6$ & $1.85 \mathrm{e}+6$ & -0.1027 & 20 & 3 \\
N7-H2-C1_P3 & $8.39 \mathrm{e}+6$ & $8.58 \mathrm{e}+6$ & -0.0221 & 4561 & 6 \\
N7_H3_C1_P4 & $8.16 \mathrm{e}+6$ & $8.41 \mathrm{e}+6$ & -0.0297 & 3936 & 9 \\
N10_H3_C1_P5 & $1.49 \mathrm{e}+7$ & $1.68 \mathrm{e}+7$ & -0.113 & 4683 & 15 \\
N10_H5_C1_P6 & $1.46 \mathrm{e}+7$ & $1.7 \mathrm{e}+7$ & -0.141 & 4086 & 60 \\
N10_H5_C2_P7 & $1.52 \mathrm{e}+7$ & $1.74 \mathrm{e}+7$ & -0.126 & 10542 & 83 \\
\hline
\end{tabular}
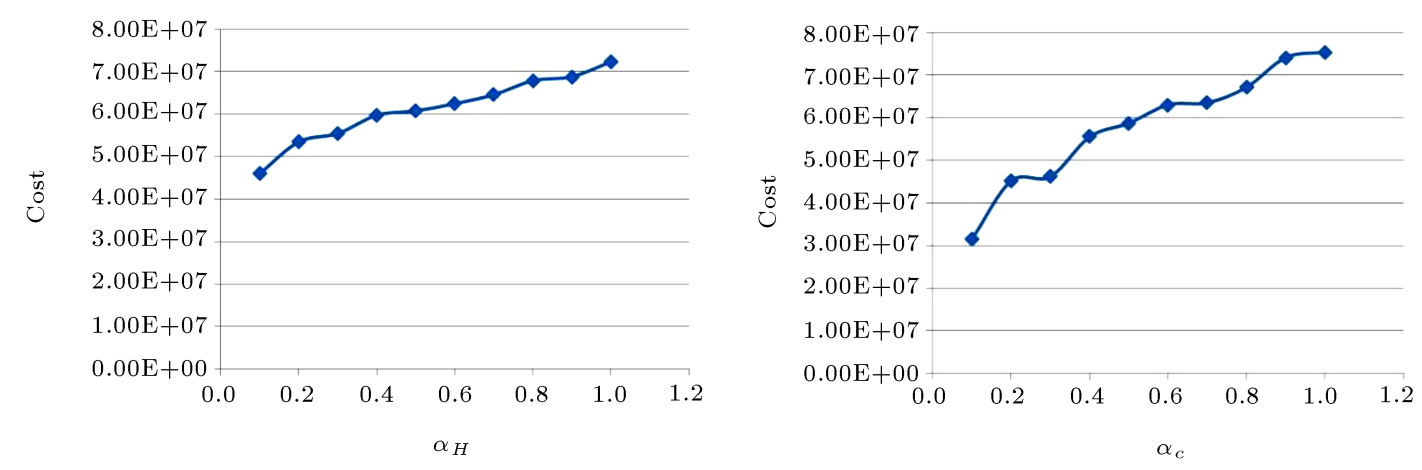

Figure 3. Sensitivity analysis of the cost function with respect to the discount factors.
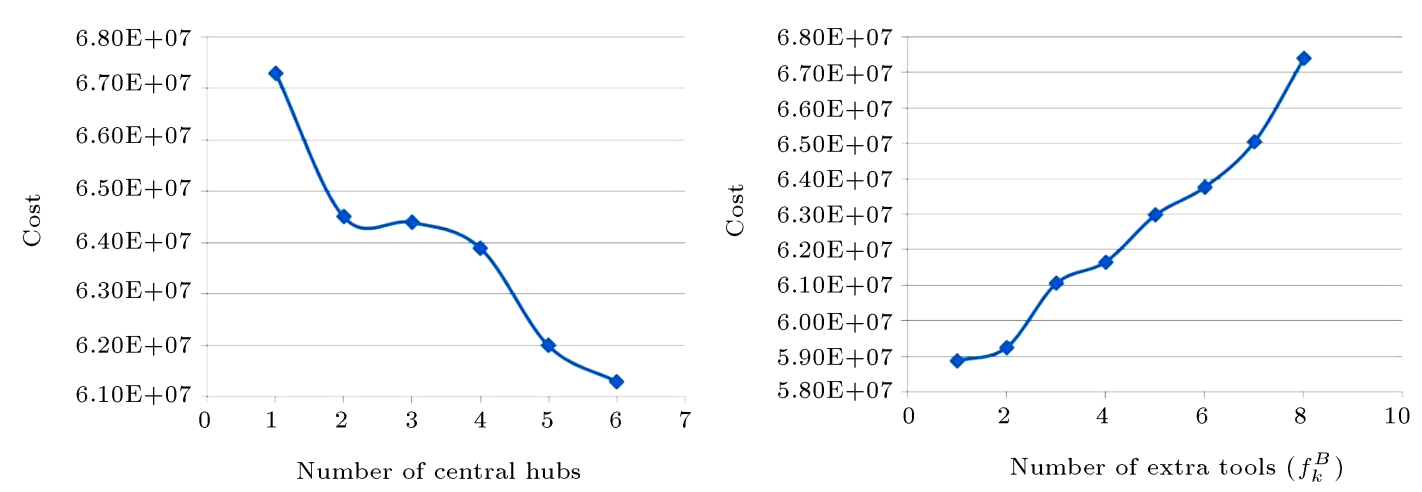

Figure 4. Sensitivity analysis of the cost function with respect to the number of central hubs and extra tools.

sum of all routing costs and costs of backup elements decreases as the number of central hubs increases. This ensures the positive role of applying central hubs in decreasing the total cost. As the number of central hubs increases in the network, the discount factors in routing between the hubs become much more profitable and, hence, the transportation costs reduce in the network; while the cost of backup units increases, the total cost also decreases.

Finally, Figure 5 shows the changes of the cost function with respect to the probability of failure due to environmental effects $(\lambda)$. As illustrated earlier, the network reliability decreases as the hub failure proba- bility increases due to environmental effects, which is reasonable because the total failure probability of the hub is directly proportional to $\lambda$.

\section{Conclusion}

In the context of hub allocation, a hierarchical hub location problem was introduced in this work as a mixed integer programming model. It can be seen that the p-hub median problem and the star p-hub median problem are two special cases of the studied problem. Specifically, if the number of central hubs is equal to that of hubs, then the problem turns into a p- 


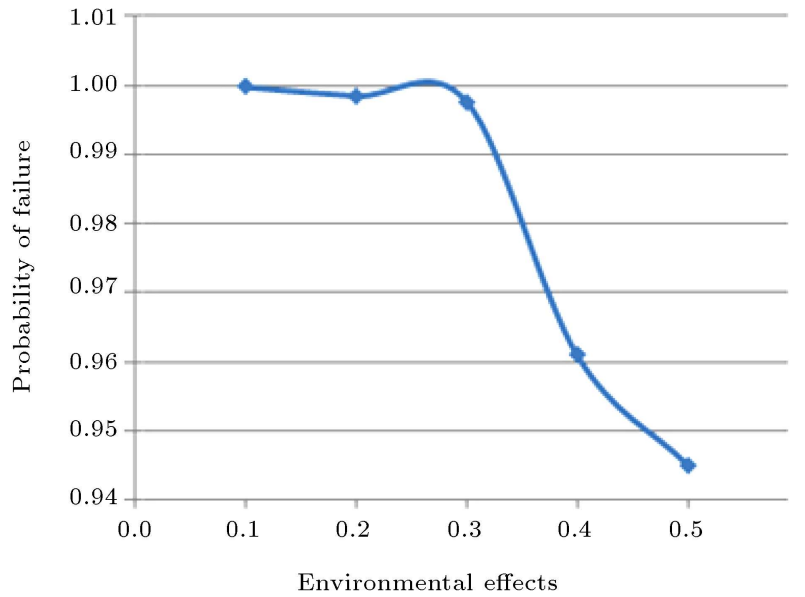

Figure 5. Sensitivity analysis with respect to $\lambda$.

hub median problem as a special case; however, if the number of central hubs to be opened is equal to 1 , then the problem turns into a star p-hub median problem, where the candidate set is singleton. By solving the developed model with the same previous instance in the literature and with the number of central hubs varying from 1 to $p$, the effect of central hubs on the total cost and total reliability was investigated.

The main model maximized the network reliability and minimized the total routing cost simultaneously. In order to provide a more realistic model, the hub failure probabilities were considered as a function of the hub's incoming traffic, while these probabilities were set to be fixed in previous studies. Reserve hub elements were also taken into account in the model to potentially increase the reliability of the network. The proposed model was then carried out in the case study with different sample size problems, the result of which was verified numerically. Meanwhile, the sensitivity analysis was performed on critical parameters and, in general, it was observed that the role of extra tools was important in increasing the total reliability; further to that, the total cost reduced by increasing the number of central hubs.

As for recommendations for future studies, some conceptual developments can be considered. For example, the reliability can be defined on arcs instead of nodes, and the model can be developed and solved again based on new conditions. In addition, other probability distribution functions can be used as failure probability instead of uniform distribution function in this study. In our model, the hub functioning probability follows the concept of geometric distribution function. This concept can be changed into more familiar distribution functions such as exponential, Weibull, and so on. On the other hand, some structural developments can be offered. For example, a budget constraint can be defined on backup establishment costs. The model is developed in deterministic condi- tions, while the model with robust conditions is closer to real-world problems. Moreover, the model can be extended to a game theory based model by considering the realistic conflicts of players.

\section{References}

1. Sim, T.K.T. "The hub covering flow problem and the stochastic p-hub center problem", PhD Thesis, University of Iowa (2007).

2. Kim, H. and O'Kelly, M.E. "Reliable p-hub location problems in telecommunication networks", Geographical Analysis, 41(3), pp. 283-306 (2009). DOI: $10.1111 / \mathrm{j} .1538-4632.2009 .00755 . \mathrm{x}$

3. Davari, S., Fazel, M.H., and Turksen, I. "The fuzzy reliable hub location problem", Fuzzy Information Processing Society (NAFIPS), 2010 Annual Meeting of the North American Fuzzy Information Processing Society: IEEE, pp. 1-6 (2010). DOI: 10.1109/NAFIPS.2010.5548271

4. Fazel, M.H., Davari, S., and Haddad Sisakht, S.A. "The Q-coverage multiple allocation hub covering problem with mandatory dispersion", Scientia Iranica, 19(3), pp. 902-911 (2012). DOI: 10.1016/j.scient.2012.03.007

5. Karimi, H. and Bashiri, M. "Hub covering location problems with different coverage types", Scientia Iranica, 18(6), pp. 1571-1578 (2011). DOI: 10.1016/j.scient.2011.09.018

6. Hamidi, M., Gholamian, M.R., and Shahanaghi, K. "Developing prevention reliability in hub location models", Journal of Risk and Reliability, 228(4), pp. 337-346 (2014). DOI: 10.1177/1748006X13519247

7. Yaman, H. "The hierarchical hub median problem with single assignment", Transportation Research Part B: Methodological, 43(6), pp. 643-58 (2009). DOI: 10.1016/j.trb.2009.01.005

8. Hamzaoui, S. and Ben-Ayed, O. "Parcel distribution timetabling problem", Operations Management Research, 4(3-4), pp. 138-149 (2011). DOI: $10.1007 / \mathrm{s} 12063-011-0056-4$

9. Yaman, H. and Elloumi, S. "Star p-hub center problem and star p-hub median problem with bounded path lengths", Computers and Operations Research, 39(11), pp. $2725-2732$ (2012). DOI: $10.1016 /$ j.cor.2012.02.005

10. Alumur, S.A., Yaman, H., and Kara, B.Y. "Hierarchical multimodal hub location problem with timedefinite deliveries", Transportation Research Part E: Logistics and Transportation Review, 48(6), pp. 11071120 (2012). DOI: $10.1016 /$ j.tre.2012.04.001

11. Davari, S. and Fazel, M.H. "The single-allocation hierarchical hub-median problem with fuzzy flows", Advances in Intelligent Systems and Computing, 195, Springer, pp. 165-181 (2013). DOI: 10.1007/978-3-64233941-7_17 
12. Fazel, M.H., Davari, S., and Haddad Sisakht, S.A. "An empirical comparison of simulated annealing and iterated local search for the hierarchical single allocation hub median location problem", Scientia Iranica, 22(3), pp. 1203-1217 (2015).

13. Zhong, W., Juan, Z., Zong, F., et al. "Hierarchical hub location model and hybrid algorithm for integration of urban and rural public transport", International Journal of Distributed Sensor Networks, 14(4) (2018). DOI: $10.1177 / 1550147718773263$

14. Smith, H., Cakebread, D., Battarra, M., et al. "Location of a hierarchy of HIV/AIDS test laboratories in an inbound hub network: case study in South Africa", Journal of the Operational Research Society, 68(9), pp. 1068-1081 (2017). DOI: 10.1057/s41274-017-0240-5

15. Sedehzadeh, S., Tavakkoli-Moghaddam, R., Baboli, A., et al. "Optimization of a multi-modal tree hub location network with transportation energy consumption: A fuzzy approach", Journal of Intelligent and Fuzzy Systems, 30(1), pp. 43-60 (2016). DOI: 10.3233/IFS151709

16. Korani, E. and Sahraeian, R. "The hierarchical hub covering problem with an innovative allocation procedure covering radiuses", Scientia Iranica, 20(6), pp. 2138-2160 (2013).

17. Li, T.-T., Song, R., He, S.-W., et al. "Optimization model of comprehensive passenger hub in urban agglomeration based on hierarchical layout", China Journal of Highway and Transport, 29(2), pp. 116-122 (2016).

18. Li, T.-T., Song, R., He, S.-W., et al. "Multiperiod hierarchical location problem of transit hub in urban agglomeration area", Mathematical Problems in Engineering, online (2017). DOI: 10.1155/2017/7189060

19. Dükkanci, O. and Kara, B.Y. "Routing and scheduling decisions in the hierarchical hub location problem", Computers and Operations Research, 85, pp. 45-57 (2017). DOI: 10.1016/j.cor.2017.03.013

20. Ryerson, M.S. and Kim, H. "Integrating airline operational practices into passenger airline hub definition", Journal of Transport Geography, 31, pp. 84-93 (2013). DOI: $10.1016 /$ j.jtrangeo.2013.05.013

21. Karimi, M., Eydi, A.R., and Korani, E. "Modeling of the capacitated single allocation hub location problem with a hierarchical approach", International Journal of Engineering, Transactions A: Basics, 27(4), pp. 573586 (2014).

22. Esmizadeh, Y. and Bashiri, M. "Applying hierarchical hub location problem on perishable good distribution systems", Joint International Symposium on The Social Impacts of Developments in Information, Manufacturing and Service Systems, Istanbul, Turkey, pp. 260-269 (2014).

23. Da Costa Fontes, F.F. and Goncalves, G. "Routing problem with pendular and cyclic service in a hierarchical structure of hub and spoke with multiple allocation of sub-hubs", International Conference on Industrial Engineering and Systems Management, Seville, Spain, pp. 561-567 (2015). DOI: 10.1109/IESM.2015.7380214

24. Mahmutogullari, A.I. and Kara, B.Y. "Hub location under competition", European Journal of Operational Research, 250(1), pp. 214-225 (2016). DOI: 10.1016/j.ejor.2015.09.008

25. Kim, J., Lee, S., and Lee, S. "An evacuation route choice model based on multi-agent simulation in order to prepare Tsunami disasters", Transportmetrica B: Transport Dynamics, 5(4), pp. 385-401 (2017). DOI: 10.1080/21680566.2016.1147002

26. Torkestani, S., Seyedhosseini, S.M., Makui, A., et al. "The reliable design of a hierarchical multi-modes transportation hub location problems (HMMTHLP) under dynamic network disruption (DND)", Computers \& Industrial Engineering, 122, pp. 39-86 (2018). DOI: $10.1016 /$ j.cie.2018.05.027

27. Cohon, J.L., Multi-Objective Programming and Planning, Chapter 6.2, 2nd Edn., Dover Publications (2013).

28. Ernest, A.T. and Krishnamoorthy, M. "Efficient algorithms for the uncapacitated single allocation-hub median problem", Location Science, 4(3), pp. 139-54 (1996). DOI: 10.1016/S0966-8349(96)00011-3

29. Schott, J.R. "Fault tolerant design using single and multicriteria genetic algorithm optimization", Master's Thesis, MIT University (1995).

\section{Biographies}

Mohsen Babashahi is an MS graduate student in School of Industrial Engineering at the Iran University of Science and Technology (IUST), Tehran, Iran. His research interests include network and reliability, facility location, simulation, and system dynamics.

Kamran Shahanaghi is an Associate Professor of Industrial Engineering at Iran University of Science and Technology, Tehran, Iran. His research interests include maintenance and reliability, multiple criteria decision-making, uncertain programming, and operations research. He has published papers in journals such as Computers \& Industrial Engineering, Expert Systems with Applications, Applied Mathematical Modelling, Engineering Failure Analysis, Journal of Manufacturing Systems, Reliability Engineering and System Safety, and IEEE Transactions on Reliability among others.

Mohammad Reza Gholamian is an Associate Professor in School of Industrial Engineering at the Iran University of Science and Technology (IUST), Tehran, Iran. He received his MS degree in Industrial Engineering from Isfahan University of Technology (IUT), Isfahan in 1998 and obtained PhD in Industrial 
Engineering from Amirkabir University of Technology (AUT), Tehran in 2005. Presently, he is a faculty member of Systems Engineering Group in School of Industrial Engineering and is actively engaged in conducting Academic, Research and Development Programs in the field of Industrial Engineering. He has contributed more than 172 research papers to many national and international journals and conferences. Besides, he has published 5 books by reputed publishers. His research interests are in the areas of inventory models, supply chain network design, and multi-criteria decision-making.

Arash Yavari is an MS graduate student in School of Industrial Engineering at the Iran University of Science and Technology (IUST), Tehran, Iran. His research interests include facility design, supply chain management, and optimization methods. 